\title{
Operator Independent Ignition Measurements
}

\author{
MOHAMMED M. KHAN and JOHN L. DE RIS \\ Measurements and Models Research \\ FM Global \\ 1151 Boston-Providence Turnpike \\ Norwood, Massachusetts 02062 USA
}

\begin{abstract}
An operator independent method for measuring the time to ignition is presented. Ignition occurs when the $2^{\text {nd }}$ derivative of mass loss vs. time curve reaches its peak, i.e., when the mass loss rate increases most rapidly due to applied heating. Prior to evaluating the $2^{\text {nd }}$ derivative one smoothes the data using the $4^{\text {th }}$ order Savitski-Golay method. The technique is demonstrated for a wide variety of polymers (PMMA, POM, FRPP, Polyisocyanurate foam, PVC, CPVC and PVDF) to examine their ignition behavior in ambient air as well as in $40 \%$ oxygen. The $2^{\text {nd }}$ derivative and the observed ignition times are compared. In all cases, the times are identical in $40 \%$ oxygen. The two times are also identical for ordinary polymers in air. However, for the halogenated fuels, PVC, CPVC and PVDF, the observed ignition is both intermittent and delayed. For halogenated fuels, enhanced ambient oxygen accelerates gas-phase combustion, but has negligible effect on initiation of mass transfer. In most cases, the agreement between observed and $2^{\text {nd }}$ derivative ignition times in air and in $40 \%$ oxygen is quite remarkable, confirming that the operator independent $2^{\text {nd }}$ derivative ignition method is both reliable and consistent.
\end{abstract}

KEYWORDS: operator independent ignition, observed ignition, mass loss rate

\section{INTRODUCTION}

In most standard ignition tests [1,2], time to piloted ignition is usually measured by visually observing the first time at which the flame remains attached for at least four seconds. Although this method tends to be reproducible for most ordinary solid polymeric materials, it is difficult for an operator to gain the necessary experience. It becomes especially difficult even for a skilled operator when the materials are highly fire retarded. When burning in air, an intermittent flame can remain for a long time above the material surface without attaching itself to the sample.

The objective of this study is to address these measurement problems by developing an operator independent measurement technique for obtaining the time to ignition. In this study, piloted ignition times are determined by the peak $2^{\text {nd }}$ derivative of the mass loss vs. time curve, i.e., at the instant when mass loss rate increases most rapidly indicating the moment of ignition. This methodology also compares the ignition in normal air and an enhanced oxygen environment to better reproduce ignition phenomena in large-scale fire where the vaporized fuel is immediately fed into the flames.

The ignition of a combustible solid, heated by an external source, starts with the solidphase thermal decomposition and evolution of combustible gases leading to gas-phase combustion, and a sustained diffusion flame. Ignition tends to occur faster in the presence of a pilot source than if the sample is allowed to ignite spontaneously. Most standard ignition tests [1,2], however, are performed in the presence of a pilot source, such as an electric spark, a small gas flame or a heated wire. The ignition and subsequent burning of 
a solid sample is sensitive to heat losses from the rear surface, especially for thermally thin samples. These heat losses depend on the sample holder and its environment, making the ignition measurements apparatus dependent. To minimize apparatus dependencies, we have designed a sample holder [3], shown in Fig. 1, that minimizes heat losses from the rear and sides of the sample being heated.

\section{EXPERIMENTAL APPROACH}

\section{Samples}

In this study, we examine a wide variety of polymeric solids (weighing $15-125 \mathrm{~g}$ ) to establish the generality of operator independent ignition technique. These include $9.5 \mathrm{~mm}$ thick clear polycast polymethylmethacrylate (PMMA) [4], gray polyvinyl chloride (PVC), ivory colored chlorinated polyvinyl chloride (CPVC), off white polyvinlidenefluoride (PVDF), black polyoxymethylene (POM), white fire retarded polypropylene (FRPP) and beige polyisocyanurate foam (51 mm thick). PMMA and POM are much studied materials having well established ignition and combustion properties. PVC-gray is a typical fire retarded PVC whose fire properties are well known. CPVC and PVDF are highly fire retarded polymers whose ignition properties are difficult to measure.

\section{Sample Preparation}

Experiments are performed in the ASTM E-2058 Fire Propagation Apparatus (FPA). Horizontal samples of diameter, $96.5 \mathrm{~mm}\left(0.00731 \mathrm{~m}^{2}\right.$ exposed surface area) are sealed (both rear and side) with $0.075 \mathrm{~mm}$ thick fiberglass adhesive aluminum tape and then mounted in a well-insulated aluminum pan ( $0.89 \mathrm{~mm}$ wall thickness). The samples in the Sample Holder are insulated with 4 layers of $3 \mathrm{~mm}$ thick Cotronics ${ }^{\circledR}$ ceramic paper [3]. See Fig. 1. The sample holders are dried in an oven for 24 hours (at $100^{\circ} \mathrm{C}$ ) and transferred to a desiccator before each experiment.

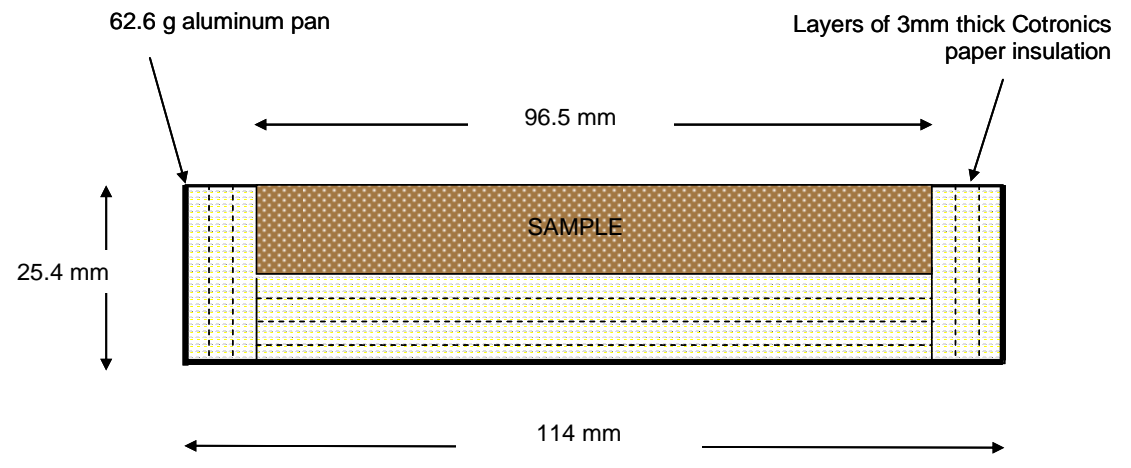

Fig. 1. Insulated sample holder.

The top surface of each sample is maintained flush with the top of the ceramic insulation. The sample surfaces are prepared with one thin coat of spectrally flat black Thurmalox ${ }^{\circledR}$ 250 high temperature paint, thereby making sure of the applied radiant energy is actually absorbed by the sample. The total mass per unit area of paint coating is $25-40 \mathrm{~g} / \mathrm{m}^{2}$. The POM, polyisocyanurate foam and FRPP samples were instead evenly coated with carbon black powder $(\sim 1 \mathrm{~g})$, because the paint might have affected the ignition measurements. 


\section{Load Cell for Mass Loss Measurements}

A load cell with a nominal accuracy of $0.1 \mathrm{~g}$ and a measuring range of $0-1000 \mathrm{~g}$ is used. The load cell noise averaged over one second has a peak-to-peak value of $20 \mathrm{mg}$. The load cell is calibrated for each set of ignition tests involving a different fuel or ambient. The output voltage from the load cell is set to zero by adjusting the tare, with the appropriate empty sample holder in position. A NIST-traceable weight corresponding to the weight of the sample to be tested is placed on the sample holder and the output voltage is measured. In order to check the linearity, this procedure is repeated with three other NIST-traceable weights including the entire sample weight range.

The data acquisition system introduced a two second delay in reporting the mass loss. This was established by dropping a small object on the load cell platform and recording the event with the data acquisition system. All data in this study are recorded times which have been corrected for this two second delay time.

\section{Ignition Measurements}

The sample holder with sample is placed horizontally on a light weight three-pronged load cell platform mount. A pre-mixed, $10 \mathrm{~mm}$ long ethylene-oxygen pilot flame is positioned about $10 \mathrm{~mm}$ above and near the center of the top surface to ensure prompt ignition if sufficient fuel vapors are present. Experiments are conducted in both normal air and in $40 \%$ oxygen both having a $3.3 \times 10^{-3} \mathrm{~m}^{3} / \mathrm{s}$ volume flow in the $162 \mathrm{~mm}$ inside diameter and $431 \mathrm{~mm}$ long quartz tube of the ASTM E-2058 apparatus.

The data acquisition system is started at the same time the radiant heaters are turned on, and then, 30 seconds later, the water-cooled radiation shield protecting the sample in the apparatus drops to expose the sample. The sample weight is recorded each second by averaging 100 readings per second. The mass transfer rate $(\mathrm{g} / \mathrm{s})$ is evaluated each second by evaluating least-squares slopes as well as from the $1^{\text {st }}$ derivative of Savitsky-Golay [5] smoothing. The detailed algorithms for evaluating the peak $2^{\text {nd }}$ derivative of the mass loss are given in the next section.

The weight loss is continuously measured during the experiments. Tests are made for different radiant heat flux exposures. The time to visual ignition is determined as the first moment that a flame remains attached for at least four seconds. At the end of each experiment, the sample from the sample holder is discarded and the sample holder is immediately transferred to a desiccator to avoid moisture absorption by the insulation.

\section{ANALYSIS TECHNIQUE}

\section{Evaluation of $2^{\text {nd }}$ Derivative}

When analyzing a time series describing transient phenomena, one frequently needs to smooth the data (i.e., suppress noise) to obtain a clearer view of the underlying transient phenomena. The need for smoothing becomes especially important when evaluating derivatives. The taking of time derivatives amplifies the higher frequency signal components containing most of the noise. A common method of smoothing data is to simply average the signal over a time window including data points just ahead and behind the point of interest. Such averaging suppresses the noise, but it can also unduly smooth out and distort the underlying signal. There is a trade-off between noise suppression and signal distortion. Too much noise suppression (smoothing) leads to signal distortion. Too 
little noise suppression prevents separation of signal from noise. There is a considerable literature on the smoothing of spectral data in the field of chemistry [5]. The literature addresses a similar problem - namely suppressing noise without distorting the magnitude and shape of the underlying spectral peaks. As shown later, the smoothing is best accomplished by least squares fitting of a fourth order polynomial to a window of data surrounding each point in the time series.

\section{Difference of Least Squares Slopes}

The taking of derivatives amplifies the higher frequency components that contain the noise. The smoothing process suppresses these higher frequency components. It helps to first suppress the noise by smoothing the data and subsequently take any needed derivatives. Perhaps the simplest method of evaluating the second derivative of noisy data is to (1) first fit linear least-squares lines to the data surrounding each data point, (2) determine their slopes, and then (3) evaluate the second derivative by taking the difference in slopes between adjacent fitted lines. This method is easy to apply and works quite well. It suppresses the noise before taking derivatives. However, the smoothing introduces considerable distortion of the second derivative. This motivates our search for a better smoothing technique.

\section{Savitski-Golay -- 4th Order}

At ignition, there is a rapid increase in the rate of sample mass loss. This creates a strong peak in the second derivative of the weight loss curve. One wishes to preserve the essential features of this strong peak. The second, third and fourth derivatives of the mass loss curve are closely associated, respectively, to the magnitude, location and width of the peak. This suggests one should use least-squares to fit a fourth order polynomial to data surrounding each data point. To suppress the inevitable experimental noise, one needs to include a significant number of extra data points in the window surrounding each point of the curve. This is known as the $4^{\text {th }}$ order Savitski-Golay smoothing technique. One simply sums the products of each surrounding data point times a coefficient depending on the size of the window and the position of the particular data point in the window. The algorithm is computationally efficient and easy to apply.

\section{An Analytic Example}

The above two noise suppression methods of are examined in Fig. 2. The smooth heavy lines show the $1^{\text {st }}$ and $2^{\text {nd }}$ derivatives for a smooth analytic mass loss curve simulating ignition. The mass loss starts at 40 seconds and completes at 70 seconds, 30 seconds later. The $2^{\text {nd }}$ derivative peaks at 52 seconds $-40 \%$ into the 30 second ignition interval. The analytic mass loss curve is evaluated each second. Gaussian noise with a standard deviation of $5 \%$ is then added to each data point. The noise at each data point is assumed statistically independent. The $1^{\text {st }}$ derivative of the noisy mass loss is shown by dotted line in Fig.2. One can now compare the smoothing and distortion produced by each technique.

The "horizontal" data before and after the ignition time interval shows that the $25 \mathrm{pt}$. window Difference in Least Squares slopes (DLS) achieves the greatest smoothing, while the 17 pt. Savitski-Golay (S-G) provides the least smoothing. On the other hand, looking at the peak $2^{\text {nd }}$ derivative the Savitski-Golay methods produce the least distortion, whereas the 25 pt. difference in least squares slopes produce the greatest distortion - as inferred from their reduction in the peak $2^{\text {nd }}$ derivative. This example indicates that $25 \mathrm{pt}$. 
Savitski-Golay smoothing technique is probably best suited for evaluating operator independent ignition.

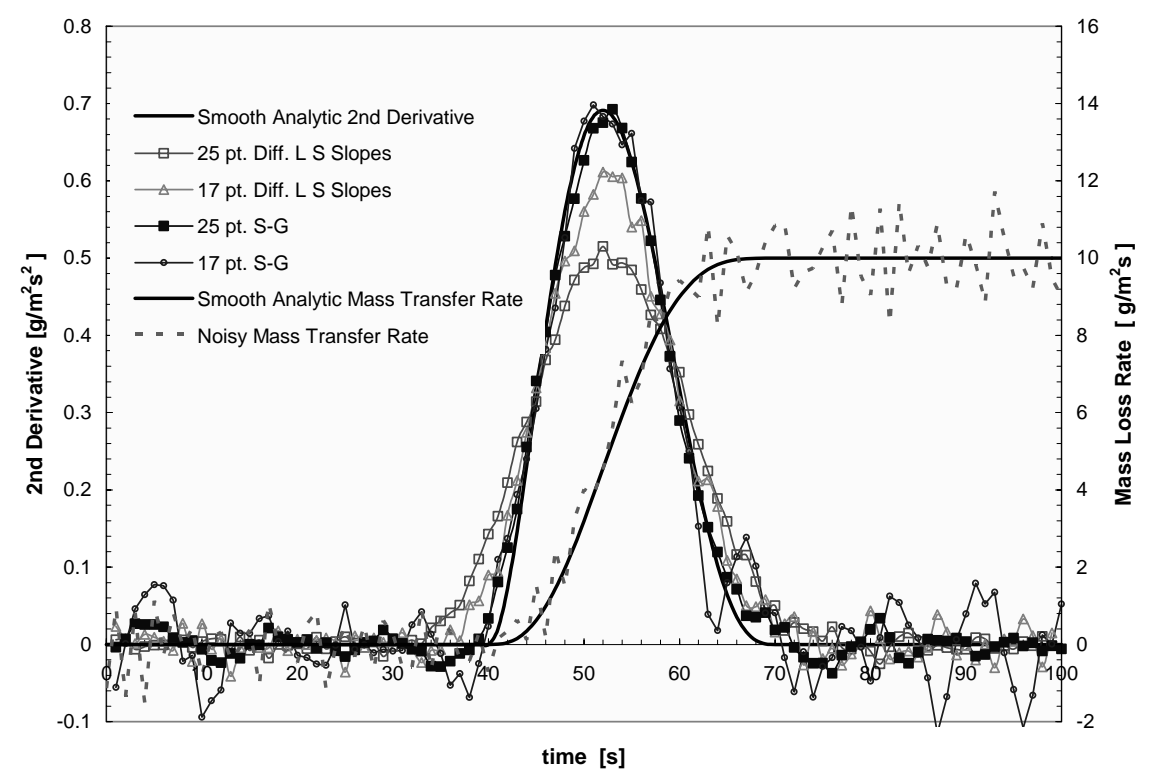

Fig. 2. Comparison of Savitski-Golay and differential slopes method for evaluating $2^{\text {nd }}$ derivative.

The distortions produced by both techniques are correlated in Fig. 3 . The ordinate is the fractional reduction from the peak $2^{\text {nd }}$ derivative of the analytic example. The abscissa is the total time interval of the window, $w \Delta \tau$ divided by the total rise time, $\tau$ of the ignition event. The individual data points are for different rise times and window sizes. The figure shows that the Savitski-Golay smoothing method produces negligible distortion for window sizes less than the ignition rise time. The DLS technique produces considerable distortion for window sizes comparable to ignition rise times.

In contrast, Fig. 4 shows the DLS technique provides superior noise rejection. The noise attenuation of 25 point S-G technique is comparable to the noise attenuation of a 17-point DLS technique. The formulae in Fig. 4 give the general correlation of the noise attenuation of each technique. Superimposed on the graph are distortion curves for each technique for ignition rise times of 20 and 30 seconds. The distortion of a 25-point S-G routine for a 30 second rise time is approximately $4 \%$; whereas the distortion of a 17 or 19 -point DLS routine having similar noise attenuation is of the order of $15 \%$. The Savitski-Golay technique is clearly superior.

For the analysis of ignition mass loss data the authors recommend using 25-point S-G smoothing for the $2^{\text {nd }}$ derivative and 19-point S-G smoothing for the $1^{\text {st }}$ derivative rate of mass loss data (which is also found to be acceptable as the 17 and 25-point S-G smoothing). 


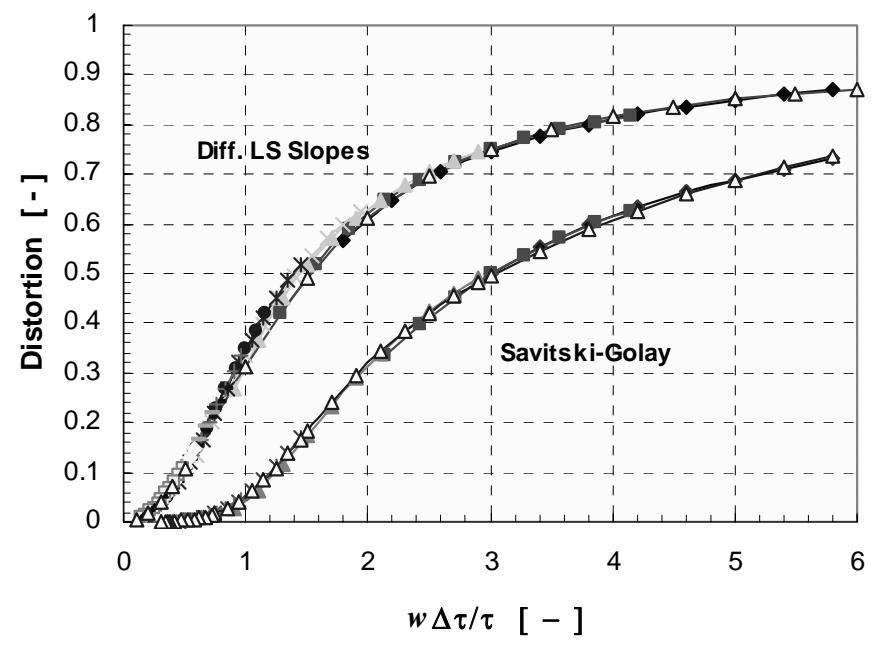

Fig. 3. Correlations of distortions produced by the difference in least square slopes and Savitski-Golay smoothing.

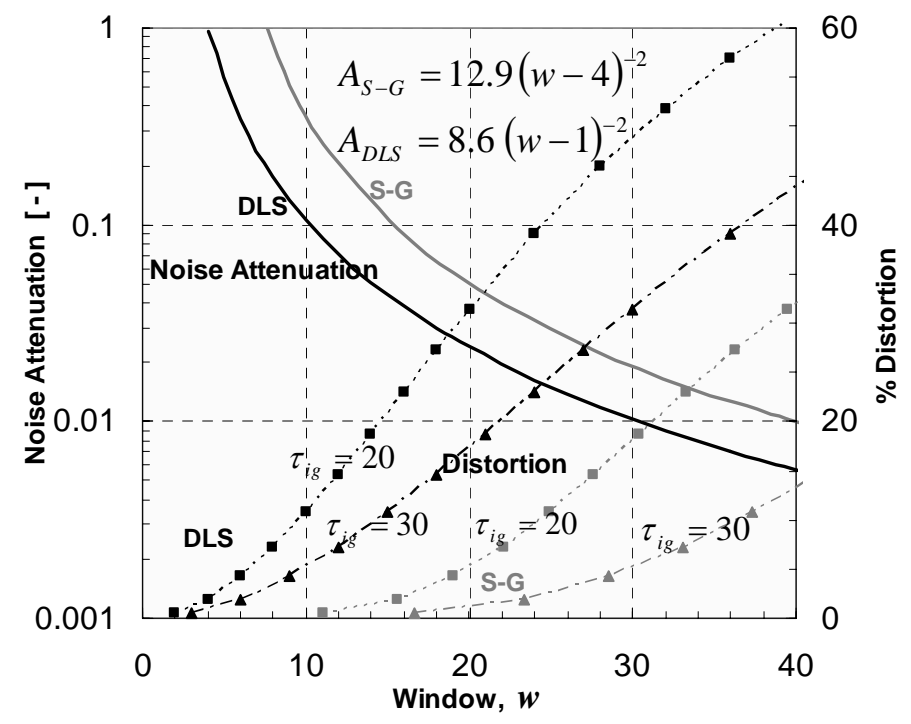

Fig. 4. Correlation of noise attenuation for Savitski-Golay and difference in least square slopes vs. number of window data point.

\section{RESULTS AND DISCUSSION}

\section{Operator Independent Ignition}

Figure 5 illustrates the Savitski-Golay technique for determining operator independent ignition. It shows the mass loss rate (the solid line curve) of PMMA sample subjected to an applied heat flux of $40-\mathrm{kW} / \mathrm{m}^{2}$. The broken line curve gives the $2^{\text {nd }}$ derivative of the mass loss vs. time using the second $y$-axis on the right hand side. The observed ignition 
occurs at the time when mass loss rate increases most rapidly. The operator independent ignition is determined at the peak $2^{\text {nd }}$ derivative of mass loss vs. time. Note that the occurrences of the two ignitions are very close.

For PMMA the combustion heat release rate $\left(\mathrm{kW} / \mathrm{m}^{2}\right)$, [i.e., mass loss rate per unit surface area $\left(\dot{m}^{\prime \prime}, \mathrm{g} / \mathrm{s}-\mathrm{m}^{2}\right)$ times the chemical heat of combustion $\left.\left(\Delta H_{c}, \mathrm{~kJ} / \mathrm{g}\right)\right]$ is about $180 \mathrm{~kW} / \mathrm{m}^{2}$. The chemical heat of combustion, as presented in Table 1 , is determined by integrating the chemical heat release rate (from ignition to flame out time) divided by the mass loss during the same time interval. For cases of no observed ignition, one may be very interested in the total potential combustion heat release rate that could add fuel to an already present exposure fire. This becomes especially important for highly fire-retarded materials, whose ignitions may not be visually observed.

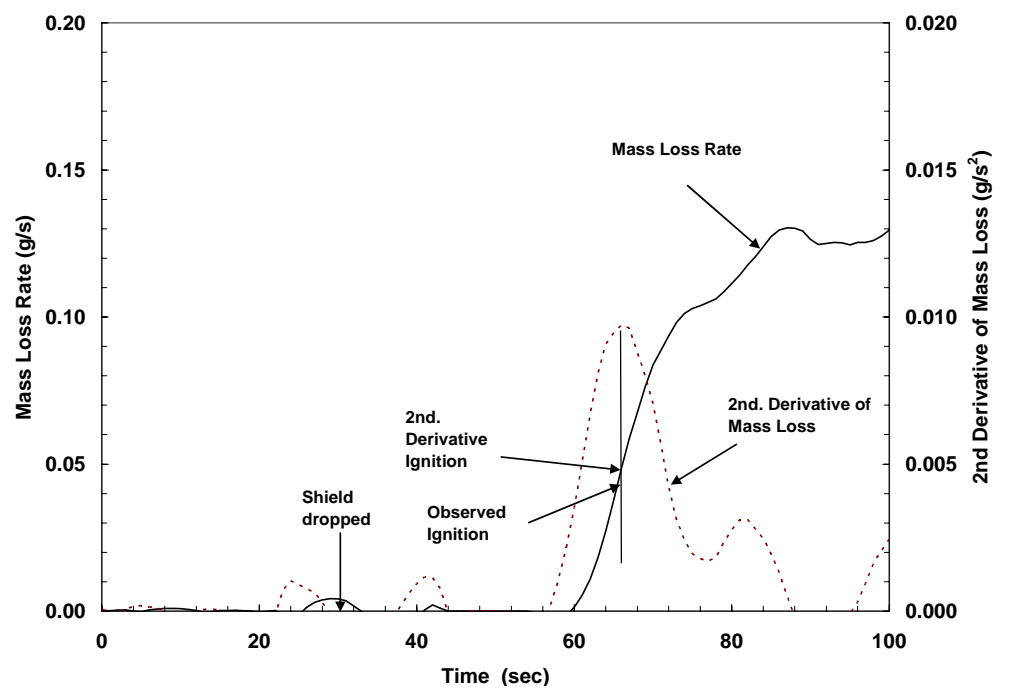

Fig. 5. Mass loss rate and $2^{\text {nd }}$ derivative of mass loss as a function of time for PMMA sample, exposed to $40 \mathrm{~kW} / \mathrm{m}^{2}$ applied heat flux in normal air with $3.3 \times 10^{-3} \mathrm{~m}^{3} / \mathrm{s}$ flow.

\section{Observed vs. $2^{\text {nd }}$ Derivative Ignition Times}

The observed and $2^{\text {nd }}$ derivative ignition times as function of applied heat flux in normal air as well as in $40 \%$ oxygen are obtained for PMMA, POM, FRPP, Polyisocyanurate foam, PVC, CPVC and PVDF using the technique as described above. All the data, when plotted by inverse square root of ignition times versus applied heat flux form a straight line implying a thermally thick response. The corresponding inverse slopes, represented by $\Delta T_{i g}\left(\pi k_{s} \rho_{s} c_{s} / 4\right)^{1 / 2}$, are presented in Table 1 . This quantity is sometimes called the Thermal Response Parameter (TRP). It is a solid phase thermal parameter associated with gas phase ignition.

The TRP determined from both the observed and $2^{\text {nd }}$ derivative ignition methods are found to be almost identical for PMMA, POM and FRPP in normal air as well as in $40 \%$ oxygen environment. This indicates that piloted ignition is typically determined by solid phase pyrolysis processes. 
We now address the gas-phase fire-retarded materials. The TRP values for Polyisocyanurate foam in normal air are very similar for observed and $2^{\text {nd }}$ derivatives. Ignition measurements in $40 \%$ oxygen were not conducted for this material.

Table 1. Correlation for thermal response parameter*.

\begin{tabular}{|c|c|c|c|c|c|}
\hline \multirow[t]{2}{*}{ Samples } & \multicolumn{2}{|c|}{$\begin{array}{c}\Delta T_{i g}\left(\pi k_{s} \rho_{s} c_{s} / 4\right)^{1 / 2} \\
\left(\mathbf{k W}-\mathbf{s}^{1 / 2} \mathbf{m}^{-2}\right) \\
2^{\text {nd }} \text { Derivative Ignition }\end{array}$} & \multicolumn{2}{|c|}{$\begin{array}{c}\Delta T_{i g}\left(\pi k_{s} \rho_{s} c_{s} / 4\right)^{1 / 2} \\
\left(\mathbf{k W}-\mathbf{s}^{1 / 2} \mathbf{m}^{-2}\right) \\
\text { Observed Ignition }\end{array}$} & \multirow[t]{2}{*}{$\Delta H_{c}(\mathbf{k J} / \mathbf{g})$} \\
\hline & $\begin{array}{c}\text { Ambient } \\
\text { Air }\end{array}$ & $\begin{array}{c}40 \% \\
\text { Oxygen } \\
\end{array}$ & $\begin{array}{c}\text { Ambient } \\
\text { Air }\end{array}$ & $\begin{array}{c}40 \% \\
\text { Oxygen } \\
\end{array}$ & \\
\hline PMMA & 254 & 245 & 239 & 230 & 22.0 \\
\hline POM & 276 & 276 & 252 & 260 & 14.4 \\
\hline FRPP & 270 & 315 & 276 & 301 & 19.5 \\
\hline $\begin{array}{c}\text { Polyisocyanurate } \\
\text { Foam }\end{array}$ & 110 & ND & 107 & ND & 13.9 \\
\hline PVC-gray & 220 & 201 & 498 & 200 & 7.9 \\
\hline CPVC & 218 & 220 & NI & 230 & 6.6 \\
\hline PVDF & $320--787$ & 321 & $447--508$ & 324 & 8.1 \\
\hline
\end{tabular}

NI: No ignition for applied heat flux up to $65 \mathrm{~kW} / \mathrm{m}^{2}$.

ND: Not determined.

$$
*\left(\frac{1}{t_{i g}}\right)^{1 / 2}=\frac{\dot{q}_{e}^{\prime \prime}-\dot{q}_{\text {loss }}^{\prime \prime}}{\Delta T_{i g}\left(\pi k_{s} \rho_{s} c_{s} / 4\right)^{1 / 2}}=\frac{\dot{q}_{e}^{\prime \prime}-\dot{q}_{\text {loss }}^{\prime \prime}}{T R P}
$$

Figure 6 presents the inverse square root of time to ignition as a function of radiant heat flux for PVC-gray. Note that the observed ignition occurs significantly later than the $2^{\text {nd }}$ derivative time in normal air. The value of TRP (see Table 1) for observed ignition times in normal air appears to be unrealistic in that the intercept of the trend line with the abscissa occurs at negative heat fluxes. The $2^{\text {nd }}$ derivative ignition values are more consistent. Their intercept occurs for positive heat fluxes. The ignition delay of PVC in normal air is controlled by the presence of gas phase chemical inhibitors and therefore, it is difficult for the operator to visually observe the ignition. An increase in ambient oxygen concentration enhances the ignition by accelerating chemical reactions in the gas phase, such that the observed and the $2^{\text {nd }}$ derivative ignition times occur simultaneously. The values of TRP (see Table 1 ) for PVC obtained from the $2^{\text {nd }}$ derivative ignition times are almost identical in normal air as well as in $40 \%$ oxygen, confirming that the operator independent $2^{\text {nd }}$ derivative ignition method is both reliable and consistent.

In the case of CPVC sample, ignition could not be observed even for the applied heat fluxes up to $65-\mathrm{kW} / \mathrm{m}^{2}$. See Table 1 as well as Fig. 7. Figure 8, presents the mass loss rate vs. time for CPVC exposed to $40-\mathrm{kW} / \mathrm{m}^{2}$ applied heat flux. Even though there was no observed ignition, the data reveals that the sample supported both ignition and 
combustion. It is clear from the figure that at the instant of $2^{\text {nd }}$ derivative ignition, the combustion heat release rate (chemical heat of combustion times the mass loss rate per unit sample surface area) is about $50 \mathrm{~kW} / \mathrm{m}^{2}$, which then rapidly rises further implying a flaming combustion. This is confirmed by the $2^{\text {nd }}$ derivative ignition in $40 \%$ oxygen (see Table 1). The agreement between the $2^{\text {nd }}$ derivative ignition times in normal air and the observed as well as the $2^{\text {nd }}$ derivative ignition times in $40 \%$ oxygen is again quite remarkable.

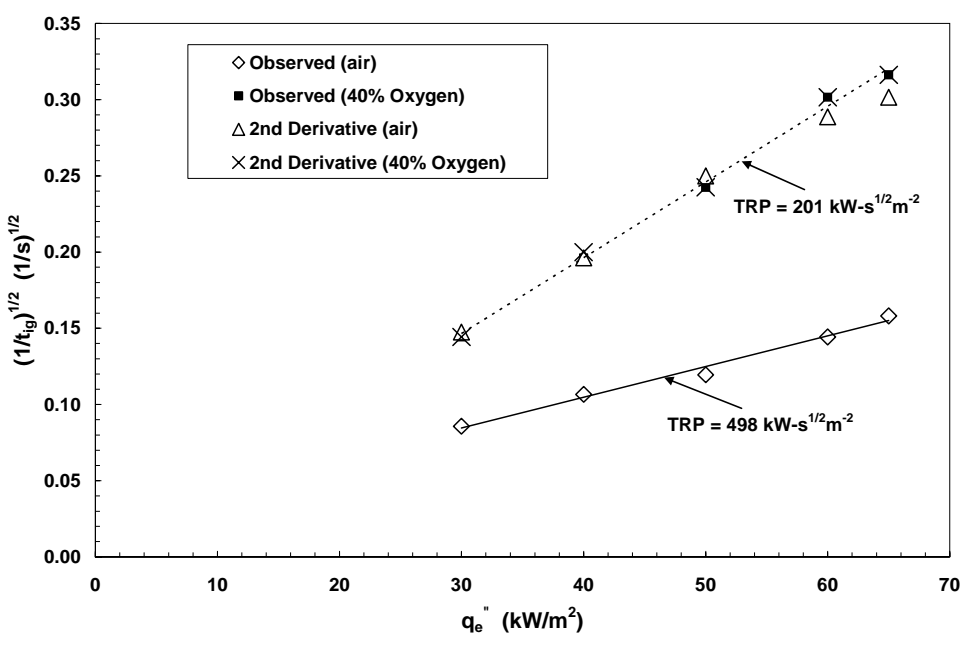

Fig. 6. Ignition time as a function of applied heat flux in normal air and $40 \%$ oxygen with $3.3 \times 10^{-3} \mathrm{~m}^{3} / \mathrm{s}$ Flow for PVC.

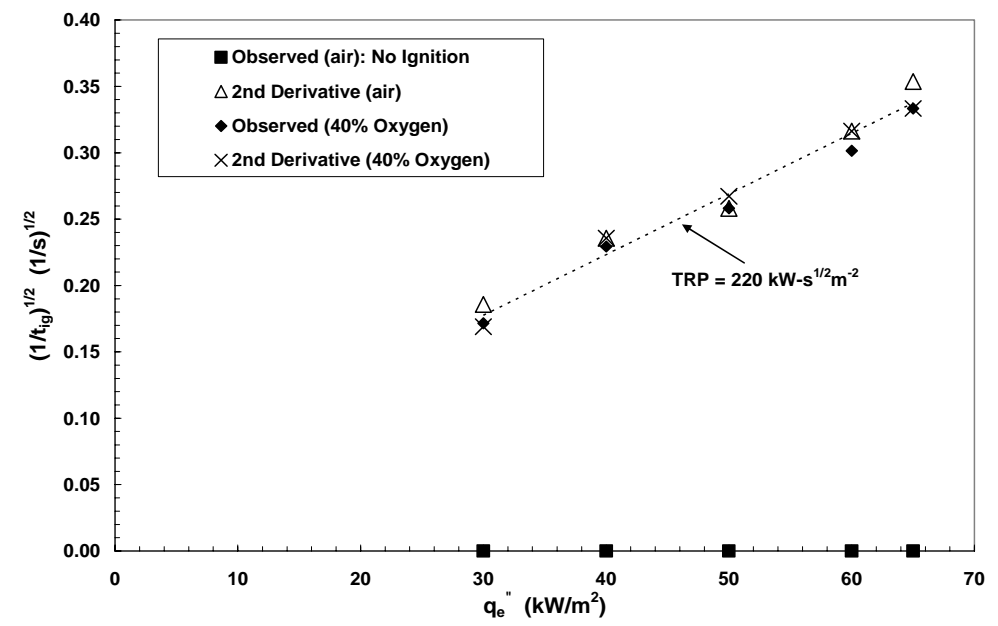

Fig. 7. Ignition time as a function of applied heat flux in normal air and $40 \%$ oxygen with $3.3 \times 10^{-3} \mathrm{~m}^{3} / \mathrm{s}$ flow for CPVC. 


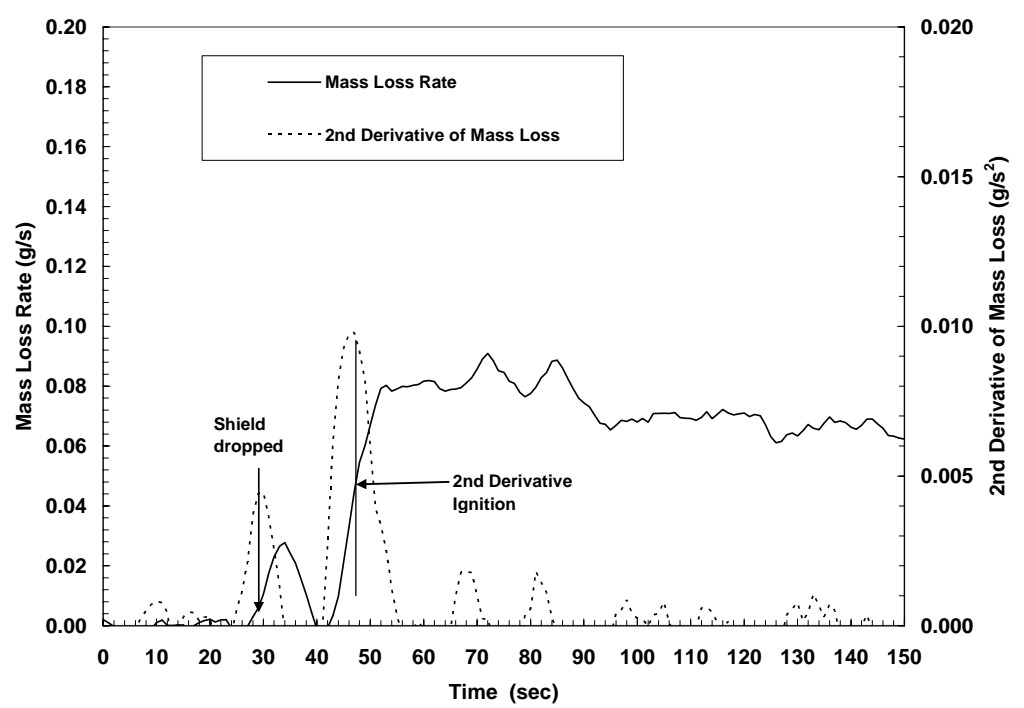

Fig. 8. Mass loss rate and $2^{\text {nd }}$ derivative of mass loss for CPVC sample exposed to $40 \mathrm{~kW} / \mathrm{m}^{2}$ applied heat flux in normal air with $3.3 \times 10^{-3} \mathrm{~m}^{3} / \mathrm{s}$ flow.

The ignition behavior of PVDF is shown in Fig. 9 as well as in Table 1. Two TRP values for observed ignition in normal air are presented in Table 1 . The ignition event is very difficult to observe for this highly fire retarded material. The first set of ignition times are observed by the operator at one location of the sample surface while the second set are when the flames have spread completely around the edge of the sample surface. Multiple peaks of the $2^{\text {nd }}$ derivative of mass loss are also found in the ignition time analysis for each applied heat flux value resulting in TRP values ranging from 787 to $320 \mathrm{~kW}-\mathrm{s}^{1 / 2} \mathrm{~m}^{-2}$.

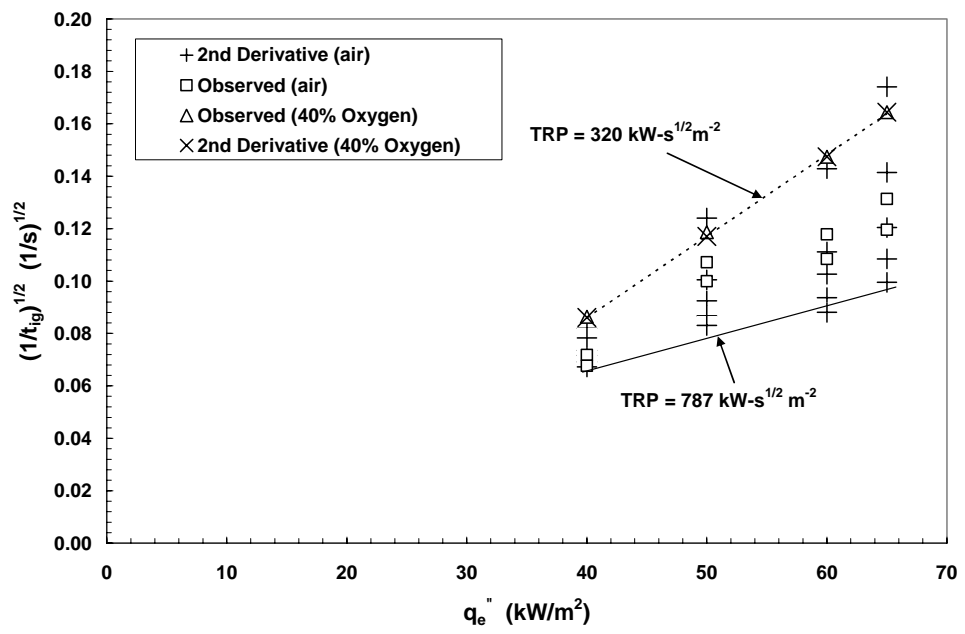

Fig. 9. Ignition time as a function of applied heat flux in normal air and $40 \%$ oxygen with $3.3 \times 10^{-3} \mathrm{~m}^{3} / \mathrm{s}$ flow for PVDF.

Figure 10 presents an example of multiple peaks of the $2^{\text {nd }}$ derivative of mass loss as well as ignitions observed by the operator, as discussed above. Due to the flickering flaming, 
it is difficult to determine the ignition times for this material by both $2^{\text {nd }}$ derivative and observed ignition methods. This is probably the reason why it is difficult for different operators to reproduce ignition time data for very highly fire-retarded materials. In $40 \%$ oxygen environment, the peak of the $2^{\text {nd }}$ derivative is very distinct and the observed and $2^{\text {nd }}$ derivative ignition data are very close as exhibited in Fig. 9.

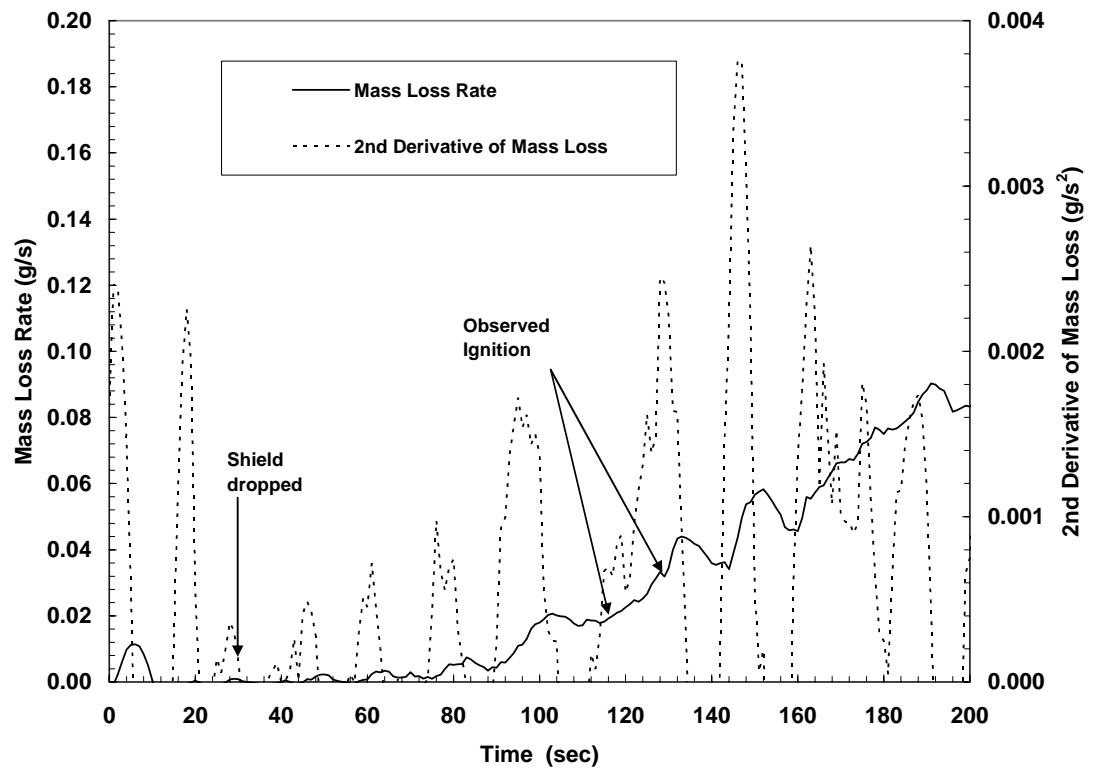

Fig. 10. Mass loss rate and $2^{\text {nd }}$ derivative of mass loss as a function of time for PVDF exposed to $50 \mathrm{~kW} / \mathrm{m}^{2}$ applied heat flux in normal air with $3.3 \times 10^{-3} \mathrm{~m}^{3} / \mathrm{s}$ flow.

The visual ignition of highly fire-retarded materials in ambient air in the laboratory is significantly delayed by the presence of gas phase inhibitors. Such delays are rarely present in actual large-scale fires where the vaporized fuel is fed immediately into the flame. The moment of release of mass at ignition having a potential heat release is the triggering event for fire growth. The ignition tests in oxygen enriched $\left(\begin{array}{lll}40 & \mathrm{O}_{2}\end{array}\right)$ environments overcome the delay due to gas phase inhibition by accelerating chemical reaction. In $40 \% \mathrm{O}_{2}$, ignition times are well defined and controlled by thermal, rather than chemical processes.

\section{CONCLUSIONS}

1. An operator independent method has been developed to determine ignition times.

2. The operator independent times are inferred from the peak $2^{\text {nd }}$ derivative of mass loss vs. time curve (i.e., when mass loss rate increases most rapidly).

3. A careful analysis shows that it is best to initially smooth the weight loss data by using the $4^{\text {th }}$ order Saviski-Golay (S-G) technique for evaluating the $2^{\text {nd }}$ derivative. It is superior to the difference in least-square method.

4. The (S-G) window size should correspond to the overall ignition time interval. The present data suggest using a 25-point S-G smoothing window for obtaining 
the $2^{\text {nd }}$ derivative and a 19 -point S-G window for the $1^{\text {st }}$ derivative, i.e., mass loss rate data.

5. The operator independent ignition method is applied here to a wide variety of polymeric solids (PMMA, POM, FRPP, Polyisocyanurate foam, PVC, CPVC and PVDF) to establish the generality of this technique. For ordinary combustibles (PMMA, POM and FRPP) the peak $2^{\text {nd }}$ derivative times closely match observed times in both ambient air and $40 \%$ oxygen.

6. For the halogenated fuels, PVC, CPVC and PVDF, observed ignition in air is both intermittent and delayed. However ignition times in $40 \%$ oxygen closely match the $2^{\text {nd }}$ derivative times in both $40 \%$ oxygen and air. The effect of enhanced ambient oxygen is to accelerate gas-phase combustion, but has negligible effect on the initiation of mass transfer. The onset of mass transfer is of principal concern for large-scale fires in that it contributes combustion energy to the fire.

7. The observed and $2^{\text {nd }}$ derivatives ignition times for fire-retarded Polyisocyanurate foam agreed in normal air. Ignition measurements in $40 \%$ oxygen were not determined for this material.

8. The agreement between observed and $2^{\text {nd }}$ derivative ignition times in normal air and in $40 \%$ oxygen is quite remarkable, confirming the operator independent $2^{\text {nd }}$ derivative ignition method as being both reliable and consistent.

\section{ACKNOWLEDGMENTS}

The authors wish to acknowledge Mr. Steve Ogden for carefully conducting experiments. We are grateful to Mr. George A. Smith and Dr. Paul A. Croce for suggesting the project and to Dr. Robert G. Bill for being actively involved throughout the course of the project.

\section{REFERENCES}

[1] ASTM E 2058-03, "Standard Test Method for Measurement of Synthetic Polymer Material Flammability Using a Fire Propagation Apparatus (FPA)," American Society for Testing and Materials, 100 Barr Harbor Drive, West Conshohocken, PA, 2002.

[2] ASTM E 1354-02, "Standard Test for Heat and Visible Smoke Release Rates for Materials and Products Using an Oxygen Consumption Calorimeter,” American Society for Testing and Materials, 100 Barr Harbor Drive, West Conshohocken, PA, 2002.

[3] de Ris, J.L., and Khan, M.M., "A Sample Holder for Determining Material Properties,” Fire and Materials, 24, pp 219-226, 2000.

[4] Khan, M.M., and de Ris, J.L., "Determination of Operator Independent Ignition for Polymeric Solids," Proceedings of the Fire and Materials 2005, $9^{\text {th }}$

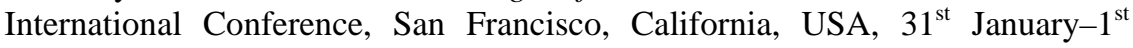
February 2005.

[5] Brereton, R.G. "Chemometrics - Data Analysis for the Laboratory and Chemical Plant” John Wiley \& Sons, 2003. 